J. Lake Sci. (湖泊科学), 2015, 27(5):767-775

DOI $10.18307 / 2015.0502$

(c) 2015 by Journal of Lake Sciences

\title{
淡水浮游植物计数与定量方法
}

\author{
钱奎梅,刘 霞, 陈宇炜** \\ (中国科学院南京地理与湖泊研究所湖泊与环境国家重点实验室,南京 210008)
}

\begin{abstract}
摘 要: 显微镜计数法是淡水浮游植物计数最常用的经典方法, 是浮游植物生物量测定的基本方法, 也是衡量其他测定 方法准确性的依据. 随着科技的发展,可见分光光度法、苂光分光光度法、流式细胞显微镜计数法、库尔特计数法等新型 细胞计数法相继问世. 对各种方法的特点进行比较, 结果表明: 光密度法、流式摄像机计数法、流式细胞仪法、叶绿素 a 法 等仅能够分析测定浮游植物生物量, 而显微镜法是测量浮游植物粒径的经典方法, 不仅可以测定浮游植物生物量, 还可 以进行浮游植物的种类及群落结构分析. 以上这些方法都可以用于浮游植物的计数与定量, 可以根据不同的需求来选择 最佳的浮游植物计数方法, 如分析方便性、样本处理速率、样本大小等. 但显微镜计数法在淡水生态学中具有不可替代的 作用.
\end{abstract}

关键词: 浮游植物; 细胞计数;生物量; 显微镜;定量

\section{A review on methods of cell enumeration and quantification of freshwater phytoplankton}

QIAN Kuimei, LIU Xia \& CHEN Yuwei

( State Key Laboratory of Lake Science and Environment, Nanjing Institute of Geography and Limnology, Chinese Academy of Sciences, Nanjing 210008, P. R. China)

\begin{abstract}
Phytoplankton cell enumeration made under a light microscope is a basic and classic method for freshwater phytoplankton cell counting and biomass calculation, and also a basis for testing the accuracy of other methods. With the development of technology, some methods on phytoplankton cell enumeration and biomass evaluation, such as visible spectrophotometry, fluorescence spectroscopy, flow microscope counting method, coulter cell counting method, have been developed. Comparing the characteristics of these methods, the results show that the optical density method, FlowCAM counting, flow cytometry, and chlorophyll-a determination method can only analyze phytoplankton biomass. As a classical method to measure the particle size of phytoplankton cell, phytoplankton analysis under a light microscope can analyze not only phytoplankton biomass, but also phytoplankton species and community structure. Since all the methods were generally appropriate for freshwater phytoplankton biomass analysis, ancillary considerations (e. g. , easiness of analysis, sample processing rate, sample size, etc. ) become critical factors for the determination of optimal phytoplankton counting method. The study indicates that cell enumeration and biomass calculation under a light microscope has an irreplaceable role in phytoplankton biomass evaluation in modern ecology.
\end{abstract}

Keywords: Phytoplankton; cell enumeration; biomass, microscope; quantification

浮游植物是一个生态学概念, 是指在水体中营浮游生活的微小植物, 通常浮游植物就是指浮游藻类,包 括蓝藻门、绿藻门、硅藻门、金藻门、黄藻门、甲藻门、隐藻门和裸藻门 8 个门类的浮游种类,已知全世界藻类 植物约有 40000 种,其中淡水藻类有 25000 种左右, 而中国已发现的淡水藻类约 9000 种 $^{[1]}$. 浮游植物是水域 生态系统的重要初级生产者,其种类组成和数量分布的生态学特征是水生生态系统的重要研究内容 ${ }^{[2]}$. 由 于浮游植物对其所处生境的水质状况反应灵敏, 能够对水体营养状态的变化迅速做出响应 ${ }^{\left[{ }^{[3]}\right.}$, 其群落结构 能够真实地反映水质状况, 是评价水体健康状况的重要指示生物 ${ }^{[4]}$.

* 江苏省博士后科研资助计划项目 (1401158C) 资助. 2014-11-20 收稿;2015-02-10 收修改稿. 钱奎梅( 1982 ), 女,博士;E-mail:qiankuimei@163.com.

** 通信作者;E-mail:ywchen@ niglas. ac. cn. 
浮游植物现存量是指某一瞬间单位水体中所存在的浮游植物的总量 ${ }^{[5]}$. 这个量用数量表示称为丰度, 用重量表示称为生物量. 由于不同水体、不同种类的藻类在个体上差异较大 (这种差异有时可达数百、数千 倍), 有些在丰度上较高的种类由于个体微小, 生物量可能低于丰度小而个体大的种类, 所以用细胞丰度来 表示生物量会过高估计细胞体积小的物种的贡献, 过低估计细胞体积大的物种的贡献, 因此, 仅用丰度就很 难全面评价不同水体浮游植物的真实情况, 而且细胞丰度对于分析浮游植物现存量在食物链和营养对策等 方面所起的作用也是有限的,应该以测算生物量为目标.

浮游植物生物量还可以通过测定叶绿素、ATP、碳含量、氮含量等方法(如化学分类方法、基于光谱的检 测方法以及分子生物学鉴定与检测技术等) 获得 ${ }^{[6-7]}$, 也可通过间接从细胞体积转化为生物量而获得. 除细 胞体积转化外, 其它方法可从不同角度获得较精确的浮游植物现存量, 但它们无法反映浮游植物的群落结 构和不同种及同种不同大小的浮游植物生物量的贡献, 从而无法解释现存量时空变化的内在原因. 只有通 过测量浮游植物细胞个体大小获得细胞体积转化后的生物量, 才能既较准确地反映浮游植物现存量, 又能 清楚地了解浮游植物的群落结构、种类组成、优势种、种类交替及不同大小细胞对浮游植物生物量的贡献.

随着科技的发展, 浮游植物细胞定量计数方法除传统的显微镜计数 ${ }^{[5]}$ 外, 可见分光光度法、苂光分光光 度法、流式细胞显微镜计数法 ${ }^{[8-10]}$ 、库尔特计数法 ${ }^{[11]}$ 等相继问世, 这些方法很多都源于海洋浮游植物分析, 可在淡水中推广应用,因此,本文对这些方法进行综述.

\section{1 浮游植物分析方法}

\section{1 基于形态学特征的分析方法}

运用光学显微镜对浮游植物进行定性、定量分析是当前鉴别和计量浮游植物最直接和可信的方法, 是 浮游植物分类鉴定的基础 ${ }^{[5,12]}$, 也是检验其他测定方法有效性的依据. 1931 年 Utermöhl 在结合 Volk 的静置 沉淀法与 Kolkwitz 的计数框基础上发明了 Utermöhl 计数法 ${ }^{[13]} .1958$ 年, Utermöhl 又规范了计数框的观测面 积 ${ }^{[14]}$. 自 1978 年联合国教科文组织 (UNESCO) 将 Utermöhl 计数法编人 《浮游植物手册》 ${ }^{[15]}$ 以来, 它在世界 范围内被接受并普遍使用, 是目前国际上浮游植物调查研究最通用的方法之一. 为减少浓缩时间, Paxinos 等 ${ }^{[16]}$ 曾改进 Utermöhl 的静置沉降浓缩为压滤浓缩, 其计数结果与传统的 Utermöhl 沉降浓缩结果具有很好 的相关性 $\left(R^{2}=0.98\right)$; 水样中不必加人鲁哥试剂进行固定, 有利于被检测的活性生物保持活性; 浓缩过程只 需 $2 \mathrm{~h}$ 左右. 为缩短浓缩时间, 王瓒等 ${ }^{[17]}$ 采用滤膜过滤方法浓缩收集水体中的藻细胞, 经超声振荡后在显微 镜下进行计数, 与传统方法的对比实验结果表明, 该方法水样用量少、操作方便、准确快捷. 膜过滤的缺点是 细胞不能用明场照明, 因为膜过滤法会扭曲图像, 并且很难识别不同物种和门类. 因此, 该方法的样本处理 速率取决于过滤的体积、样品过滤的比例以及样品中细胞浓度和杂质的量等 ${ }^{[18]}$.

由于藻类无法直接称重, 而藻类细胞形态较为规则, 且细胞比重接近于 1 , 故可用形态相似的几何体积 直接换算为生物量 (湿重) ${ }^{[19-21]}$, 即生物量为浮游植物的丰度乘以各自的平均体积, 单位为 $\mathrm{mg} / \mathrm{L}$. 单细胞的 生物量主要根据浮游植物个体形状测量分析. 因浮游植物体积在不同地区、不同季节都有较大变化,最好对 其进行直接测量, 对于数量多或体积大的种类, 特别是优势种, 更应实际测量. 测量时应根据藻类体型, 按最 近似的几何图形测量其长度、宽度、直径等, 分别求得平均值, 然后按求积公式计算出体积. 有的种类形状较 特殊, 可分解为几部分, 分别按相似几何图形求算相加. 显微镜法耗时较长, 对专业知识要求较高, 载玻片和 盖玻片的间距使细胞呈现出多种形态, 水样中的非藻颗粒物也可对样品分析造成干扰 ${ }^{[22]}$, 同时, 显微镜放大 倍数的局限可造成微型及超微型浮游植物的漏检. 但由于显微镜计数法观测面积大、准确度高、变异系数 小, 能较为全面地反映样品的种类分布情况, 目前仍然为国际上浮游植物计数最通用的方法. 该方法适用于 浓度低、种类多的样品 ${ }^{[23]}$.

血球计数法 ${ }^{[24]}$ 虽然方便、快捷, 但是如果计数室内有杂物或未洗净的微生物时, 会影响观察和计数结 果. 因此, 使用血球计数器之后必须将其清洗干净. 该方法适合于实验室纯种培养的高浓度样品. 库尔特计 数 ${ }^{[11]}$ 是通过测量细胞体积和个数, 得出各颗粒的大小, 统计出粒度的分布, 其重现分析功能可分析到单个脉 冲信号, 灵敏度较高, 该方法适合于实验室纯种培养的样品.

基于形态学特征对藻种进行鉴定的另一个重要研究方向就是利用计算机辅助完成的图像分析和识别 
技术. 目前广泛使用的流式摄像机 (flow cytometer and microscope,FlowCAM) 综合了显微镜法、叶绿素苂光法 和流式细胞仪法的功能, 能够给出每一种浮游植物的尺寸和图像; 可以对浮游植物自动计数, 并对其图像进 行处理, 经图库比对后对浮游植物进行分类测量和识别; 可以根据浮游植物形态及光学特性, 自动识别部分 特征性浮游植物, 能用于大量样品的监测分析; 可以连续 $24 \mathrm{~h}$ 自动在线监测或巡航监测; 非常适合检测基于 天然叶绿素苂光的浮游植物样品, 具有快速识别和计数的特点 ${ }^{[25-29]}$. FlowCAM 为藻类分类鉴定提供了良好 的图像及数据, 可进行种类鉴定的藻类直径应不小于 $5 \mu \mathrm{m}$, FlowCAM 要求样品浓度不能太低, 否则仪器进 样时可能会因藻类混合不匀而导致较大的误差 ${ }^{[30-31]}$. 由于浮游植物种类繁多, 不同种类的藻细胞可能在形 态结构上具有很大的相似性,同一种类在不同生长时期或以不同视角所捕获的照片在形态学上也可能有较 大的差异,使鉴别的准确度大大降低. 此外, 技术上的限制可能使获取的照片分辨率较低,增大了准确识别 的难度. 目前真正实现自动识别的系统并不多, 可识别的物种也比较少 ${ }^{[8-10,32]}$.

\section{2 基于色素的化学分析方法}

基于色素的化学分析方法是利用浮游植物不同类群间色素组成和含量的不同来确定群落组成和丰度. 其中, 优势比较明显的是高效液相色谱 (HPLC) 技术 ${ }^{[33]}$,一次操作可以分离 50 多种色素 ${ }^{[34]}$. 二极管阵列检 测器的使用使色素检测更加准确、便捷 ${ }^{[35]}$. 基于 HPLC 的光合色素分析技术能够有效地进行大量的现场样 品分析, 即使在样品处理中存在过滤产生损失或处理过程导致样品破坏的情况, 但从 HPLC 的光合色素产生 信号峰的差异仍然能辨析出其类群组成. 在野外样品检测方面, 原来因为显微计数中样品过滤或预处理所 造成的种类缺失, 现在通过 HPLC 色素峰也能清楚地辨别出来 ${ }^{[36]}$. 此外, HPLC 方法还使每个航次分析几百 个样品成为可能 ${ }^{[37]}$. 目前, 该方法仅局限在纲一级水平, 如果要进行种属水平的鉴定, 还需要寻找新的特征 色素或色素组合 ${ }^{[38-39]}$. 某些形态上相似的种类, 可能在光合色素等生物标志物上存在差异, 从而可以有效地 鉴定 ${ }^{[00]}$. 应用光合色素作为分类技术手段的缺点是尚不能完全实现属和种之间的准确分类. 此外,环境变化 以及不同的细胞生长周期也会导致浮游植物的光合色素含量发生一些变化, 从而导致分类的结果受到影 响 ${ }^{[41]}$. 所以,应用该方法对浮游植物进行定性和定量分析还很难实现.

\section{3 基于光学特性的分析方法}

借助光学特性对浮游植物进行检测的方法主要有活体苂光分析技术 ${ }^{[42]}$ 以及基于现场表观光谱和固有 光谱的浮游植物检测技术 ${ }^{[4345]}$. 苂光测定技术不需破碎细胞, 不伤害生物体, 因此通过研究叶绿素苂光来间 接研究光合作用的变化是一种简便、快捷、可靠的方法 ${ }^{[46-49]}$. 现场苂光分析法主要集中于海水样品和蓝藻监 测中对光合色素的检测 ${ }^{[47.50]}$. 可以区分浮游植物种类的便携式激光诱导苂光系统已经研制成功 ${ }^{[51]}$. 此外, 由 于不同种类浮游植物光合色素含量和组成有一定差别, 因此还可以根据吸收光谱图的差异区分不同植物类 群 ${ }^{[52]}$. 现场苂光检测法的优势在于它可以对水下灯现场环境进行快速、连续、大面积测定,但不能实现对浮 游植物的分类. 借助现场表观光谱和固有 (反射、散射、吸收) 光谱的浮游植物检测 方 $^{\text {法 }}{ }^{[53-54]}$ 简单且容易实 现, 但是探测精度受环境条件限制, 对浮游植物的识别也比较粗精. 研究认为与藻细胞粒径、色素结构有关 的色素综合效应是造成浮游植物比吸收系数变化的重要原因, 同时也影响着浮游植物吸收系数的光谱形 状 ${ }^{[55]}$. 因此, 基于水色遥感的浮游植物总生物量反演 ${ }^{[56-62]}$ 能实现对水域的大面积监测, 提供浮游植物总量信 息, 但不能对种类组成进行鉴定. 叶绿素 a 浓度测定法能够确定细胞生物量,准确度高, 但操作较复杂、耗时 长. 不同种类浮游植物的叶绿素 $\mathrm{a}$ 相对浓度随浮游植物群落的变化而变化, 同时在不同环境条件下的叶绿 素 $\mathrm{a}$ 相对浓度也有所不同 ${ }^{[63]}$. 在纯种藻培养中, 叶绿素 $\mathrm{a}$ 与细胞浓度的比率随细胞生理学和藻类生长阶段 而变化 ${ }^{[64]}$. 因此, 叶绿素 a 浓度只是浮游植物浓度的近似值.

\section{4 基于免疫分析的检测技术}

基于免疫学分析的检测方法已有几十年的历史. 早期人们只是将其作为一种常规分类学手段的补充方 法, 能准确识别的种类也不多. 近 20 年来, 随着科学技术的突飞猛进, 基于免疫分析的检测技术也有了长足 发展. 应用抗体探针的免疫测定主要有免疫苂光分析 ${ }^{[65]}$ 、酶联免疫吸附分析 ( enzyme-linked immune-sorbent assay, ELISA $)^{[66]}$ 及流式细胞免疫分析 ${ }^{[67]}$ 等. 免疫分析方法无需对样品进行预处理, 设备普及度高, 易实现 商品化. 但它需要制备高度专一的特异性抗体, 尤其在对同一种属进行区分的时候需要制备单克隆抗体,但 是传统的制备单克隆抗体的方法——杂交瘤细胞技术的费用较高且操作繁琐, 对特异性抗体的研发造成了 
一定的限制. 目前, 制备特异性强的多克隆抗体已成为可行的替代方法. 目前已有关于藻类多抗制备和特异 性鉴定的报道, 并已成功制备出多种高特异性的多克隆抗体. 将多克隆抗体技术与适当的免疫学检测方法 相结合可以实现对目标藻的定性和定量分析 ${ }^{[68-69]}$.

\section{5 基于核酸分析的检测技术}

目前基于核酸的浮游植物定性定量分析方法主要有功能基因研究、分子指纹分析技术、分子探针技术等. 功能基因的研究主要包括核糖体基因 (ribosomal RNA gene, rDNA)、细胞色素 $\mathrm{b}$ 基因 (Cytoclme b, cyt b)、增 殖细胞核抗原基因 (PCNA)、Hsp90 (热休克蛋白) 基因、质体 $r b c \mathrm{~L}$ (二磷酸核酮糖羧化酶大亚基基因)等的研 究. 其中, rDNA 凭借其为细胞所共有、功能同源且最为古老的独特优点, 成为目前应用最广泛的分子指 标 ${ }^{[70-72]}$. 虽然 rDNA 序列分析是对浮游植物定性、定量检测的一种较为可靠的方法, 但其耗时费力, 不太适合 常规测定, 而且, 用于序列比对的美国国立生物技术研究中心数据库 (national center for biotechnology information, NCBI) 里收录的序列在研究者上传后并未经过严格的审核和验证, 其准确性无法得到保证. 同时, 对 某一种类来说序列信息往往呈片段化,缺乏完整的 rDNA 基因序列信息,这就给序列比对的准确度造成了一 定的影响 ${ }^{[70-72]}$.

基于 DNA 多态性的分子指纹分析技术方法简单, 重现性好, 无需测序, 在藻类的系统比较和分类鉴定 中起着重要作用. 但是, 藻类基因组数据库的不完整性限制了这些方法的应用 ${ }^{[73]}$.

由于分子探针技术具有专一性强、准确、快速等特点, 在分子生物学研究中得到了广泛应用 ${ }^{[74-75]}$. 近年 来, 苂光原位杂交 (fluorescence in situ hybridization, FISH) 技术凭借其分析速度快和灵敏性高等优点在藻类 检测和生态学研究等领域得到广泛应用 ${ }^{[76]}$. FISH 的优势在于能准确识别出样品中的目标生物, 还能对群落 结构进行研究. 同时, 样品的全细胞形态为验证探针检测结果提供了一个重要补充. 不过, 该方法也存在一 定缺陷, 如对链状形式聚生的细胞 (拟菱形藻 Pseudonitzschia、骨条藻 Skeletonema、赤潮异弯藻 Heterosigmaak ashiwo 等) 以及在保存时容易聚集的细胞鉴定、计数较难, 对裸甲藻等一些甲板不明显微藻全细胞形态的固 定和保存也有一定难度, 还有其它一些因素也可能影响着苂光原位杂交的效果, 如固定方法、脱色条件、低 温保存以及 RNA 降解等 ${ }^{[77-78]}$.

实时苂光 PCR 不仅具有普通 PCR 的高灵敏度, 而且应用了苂光探针, 既增加了检测特异性, 又可以通 过光电传导系统直接探测 PCR 过程中苂光信号的变化, 因此还具有 DNA 杂交的高特异性和光谱技术的高 精确性. 实时荧光定量 PCR 技术不仅实现了对核酸模板的定量, 而且具有灵敏度和特异性高、能实现多重反 应、自动化程度高、无污染、实时和准确等特点. 因此, 实时荧光 PCR 也被运用到藻类学研究中 ${ }^{[79-80]}$. 但在浮 游藻类定性定量检测方面, 该技术尚处于起步阶段, 其最大的限制在于很难获得高纯度的 DNA 样品.

夹心杂交技术无需进行样品纯化、恒温杂交、操作简单. 不过, 该技术存在的致命缺陷是, 它直接检测极 易降解的 RNA, 而夹心杂交中包含抗原抗体反应和许多洗涤操作, 增加了 RNA 降解的机会, 使分析结果的 准确度和稳定性不理想. 此外, 反应中缺少温度控制也给特异性探针的设计带来很大难度. 为了克服夹心杂 交技术的缺点, 有研究者 ${ }^{[81-82]}$ 将酶保护分析方法与夹心杂交方法相结合, 采用双特异分子探针技术并将其 应用于浮游植物的检测, 但也只能对样品中十余种藻类进行定性、定量分析.

\section{2 淡水浮游植物计数及定量方法比较}

能够分析计算浮游植物总生物量的方法有显微镜法、光密度法、FlowCAM 计数法、流式细胞仪法、叶绿 素 a 法等 (表 1). 目前很多研究者 ${ }^{[83-88}$ 探讨了不同计数方法衡量某一种类微藻生物量的可行性, 结果表明, 分光光度法和荧光法是相对简便、快捷、准确的分析方法. 然而, 由于不同种类微藻细胞个体大小不同, 运动 能力不同, 而且细胞生长过程中可能存在着某些物质浓度 (如叶绿素、苂光物质等) 的变化, 而上述研究只针 对特定种类取某一生长期培养液进行稀释测定, 因此实验结果适用范围存在一定的局限性.

浮游植物计数的方法中分光光度法和库尔特计数法操作简单、耗时短, 测定结果重现性好、误差小, 测 定时可以不破坏样品, 特别适于连续测定, 是比较简便、快捷、有效的测定方法. 但分光光度法计数细胞若采 用叶绿素的最大吸收波长, 其测定容易受细胞生长和生理状态的影响, 而采用浊度比色又受细胞生长后期 细胞碎片和有机颗粒等非细胞因素的影响 ${ }^{[89]}$. FlowCAM 计数法虽然准确, 号称 “小流式细胞计数”, 但操作 
比较繁琐、耗时长, 而且数据后期处理需经过 2 次校正. 仪器计数要求细胞条件较高, 最好是处在指数生长 阶段中的细胞, 而人为计数 (如显微镜和 FlowCAM) 能分辨细胞生长后期的细胞碎片甚至死细胞. 库尔特计 数采用小孔电阻原理, 测量颗粒的大小和数量, 统计出粒度的分布, 其重现分析功能可分析到单个脉冲信 号,同时分析不同类型细胞相当方便; 其灵敏度较高,但线性范围较小 ${ }^{[90]}$. 因此, 当藻密度较小时或实验室纯 种培养、浓度高、种类单一时,采用库尔特法效果较好; 当藻密度较大时, 可采用可见分光光度法 ${ }^{[89]}$. 库尔特 计数结果一般会高于显微镜计数结果, 因其会计数一些非细胞形态的颗粒,但校正后准确度仍然较高. 在显 微镜计数以外的计数方法中, 库尔特计数法较好, 其次为分光光度计数法和 FlowCAM 计数法 ${ }^{[88]}$. 库尔特法 是一种相对简便、快捷和可靠的细胞计数方法. 流式细胞仪法使单样品操作时间较短, 能灵敏检测细胞死/ 活状态, 测定梯度配比的热处理致死细胞的结果与配比值吻合良好, 该方法用于紫外消毒处理水样中的藻 细胞计数及死/活状态分析灵敏有效. 显微镜计数法不仅能较为全面地反映样品的种类分布情况, 而且准确 度高、变异系数小, 目前仍然为国际上浮游植物调查研究最通用的方法.

\section{表 1 各种浮游植物计数和定量分析方法的优缺点比较}

Tab. 1 The advantages and disadvantages of different methods on freshwater phytoplankton cell enumeration and biomass evaluation

\begin{tabular}{|c|c|c|}
\hline 方法 & 优点 & 缺点 \\
\hline 显微镜计数法 & $\begin{array}{l}\text { 准确度高且能较为全面地反映样品的种类 } \\
\text { 分布情况. }\end{array}$ & $\begin{array}{l}\text { 耗时过长,一般至少需 } 24 \mathrm{~h} \text {; 水样分析难度大, 对专业 } \\
\text { 知识要求较高, 分析进度缓慢; 水样中的非藻颗粒物 } \\
\text { 对样品分析造成干扰. }\end{array}$ \\
\hline 血球计数法 & 方便、快捷. & $\begin{array}{l}\text { 适用于个体较大的细胞或颗粒; 必须有血球计数器才 } \\
\text { 能计数;清洗工作较为复杂,否则会增加误差. }\end{array}$ \\
\hline FlowCAM 计数法 & 操作时间短, 能灵敏检测细胞死/活状态. & $\begin{array}{l}\text { 由于藻细胞形态、结构上的相似性及一些技术上的限 } \\
\text { 制, 藻类细胞鉴别的准确度较低. }\end{array}$ \\
\hline 高效液相色谱技术 & $\begin{array}{l}\text { 准确、便捷;可以确定浮游植物群落组成和 } \\
\text { 丰度. }\end{array}$ & $\begin{array}{l}\text { 很难准确实现浮游植物的定性和定量分析; 在实际研 } \\
\text { 究中,藻类特征色素基础性数据还比较缺乏. }\end{array}$ \\
\hline 库尔特计数法 & $\begin{array}{l}\text { 不但能准确测量粒径分布, 还能测量绝对数 } \\
\text { 量和浓度. }\end{array}$ & 不能进行种类鉴定. \\
\hline 活体苂光分析技术 & $\begin{array}{l}\text { 荧光测定技术不需破碎细胞, 不伤害生物 } \\
\text { 体,还可以根据吸收光谱图的差异区分不同 } \\
\text { 浮游植物类群. }\end{array}$ & $\begin{array}{l}\text { 对浮游植物的分类较为粗糙, 易受色素组成和含量变 } \\
\text { 化的影响. }\end{array}$ \\
\hline $\begin{array}{l}\text { 基于表观光谱和固 } \\
\text { 有光谱的检测技术 }\end{array}$ & $\begin{array}{l}\text { 简单且容易实现; 能实现对水域的大面积 } \\
\text { 监测. }\end{array}$ & $\begin{array}{l}\text { 由于探测精度受环境条件限制, 对浮游植物的识别也 } \\
\text { 比较粗粘; 不能对浮游植物种类组成或类群进行鉴定. }\end{array}$ \\
\hline $\begin{array}{l}\text { 基于免疫分析的检 } \\
\text { 测技术 }\end{array}$ & 对浮游藻类的定性和(或) 定量检测. & $\begin{array}{l}\text { 作为一种常规分类学手段的补充方法来鉴定浮游植 } \\
\text { 物,能准确识别的种类不多. }\end{array}$ \\
\hline 叶绿素菼光法 & $\begin{array}{l}\text { 具有较高的稳定性和精度; 不需破碎细胞, } \\
\text { 不伤害生物体;简便、快捷. }\end{array}$ & $\begin{array}{l}\text { 对浮游植物分类较为粗糙, 易受色素组成和含量变化 } \\
\text { 的影响. }\end{array}$ \\
\hline 叶绿素 a 测定法 & 准确度高、人为误差小. & 操作较复杂、耗时长;无法进行浮游植物种类鉴定. \\
\hline 功能基因研究 & $\begin{array}{l}\text { rDNA 序列分析是对浮游植物定性、定量检 } \\
\text { 测的一种较为可靠的方法. }\end{array}$ & 耗时费力, 不太适合常规测定; 准确性无法得到保证. \\
\hline 分子指纹分析技术 & $\begin{array}{l}\text { 基于 DNA 多态性的分子指纹分析技术,方 } \\
\text { 法简单,重现性好,无需测序,在藻类的系统 } \\
\text { 比较和分类鉴定中起着重要作用. }\end{array}$ & 藻类基因组数据库的不完整性限制了其应用. \\
\hline 分子探针技术 & $\begin{array}{l}\text { 分析速度快、灵敏性高; 能对群落结构进行 } \\
\text { 研究. }\end{array}$ & 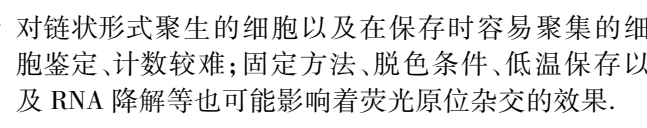 \\
\hline $\begin{array}{l}\text { 实时若光定量 PCR } \\
\text { 检测法 }\end{array}$ & $\begin{array}{l}\text { 灵敏度和特异性高; 能实现多重反应; 自动 } \\
\text { 化程度高; 无污染; 实时、准确. }\end{array}$ & $\begin{array}{l}\text { 在浮游藻定性、定量检测方面,该技术尚处于起步阶 } \\
\text { 段,其最大的限制在于很难获得高纯度的 DNA 样品. }\end{array}$ \\
\hline
\end{tabular}


浮游植物种类及群落结构分析对于深人研究淡水浮游植物生理特性、群落结构与功能、在淡水生态系 统中的作用等都具有重要的科学意义. 目前显微镜计数法 ${ }^{[91-92]}$ 、库尔特计数法 ${ }^{[11]}$ 、流式细胞仪法 ${ }^{[93-94]}$ 等均 可测量水体浮游植物粒径. 库尔特计数法虽然简单快速, 可以方便地测量其粒径分布, 但不能进行种类鉴 定. 显微镜计数法是测量浮游植物粒径的经典方法, 具有直观、准确等优点, 并可进行种类鉴定 ${ }^{[95]}$. 能够鉴定 浮游植物种类的藻类计数法有显微镜法、PCR 法, 而 PCR 法在浮游藻定性、定量检测方面尚处于起步阶段, 其最大的限制在于很难获得高纯度的 DNA 样品 ${ }^{[96]}$.

选择适当的浮游植物计数方法对浮游植物的相关研究十分重要. 以上这些方法都可以用于浮游植物的 计数与定量, 可以根据不同的需求来选择最佳计数方法, 如样本大小、便于分析、样本处理率等. 显微镜细胞 定量计数法作为浮游植物定量和计数最经典的方法, 是衡量浮游植物定量、计数及其它方法是否准确的标 准,在淡水生态学中具有不可替代的作用.

\section{3 参考文献}

[ 1 ] 魏印心, 胡鸿钧. 中国淡水藻类——系统、分类及生态. 北京: 科学出版社, 2006:1023.

[ 2 ] Watson SB, Mccauley E, Downing JA. Patterns in phytoplankton taxonomic composition across temperate lakes of differing nutrient status. American Society of Limnology and Oceanography, 1997, 42(3) : 487-495.

[ 3 ] Suikkanen S, Laamanen M, Huttunen M. Long-term changes in summer phytoplankton communities of the open northern Baltic Sea. Estuarine, Coastal and Shelf Science, 2007, 71 : 580-592.

[ 4 ] Stevenson JR, Peterson CG, Kirschtel DB et al. Density-dependent growth, ecological strategies, and effects of nutrients and shading on benthic diatom succession in streams. Journal of Phycology, 1991, 27(1) :59-69.

[ 5 ] 章宗涉, 黄祥飞. 淡水浮游生物研究方法. 北京:科学出版社, 1991:426.

[6 ] Boyce DG, Lewis MR, Worm B. Global phytoplankton decline over the past century. Nature, 2010, 466: 591-596.

[ 7 ] Welschmeyer NA. Fluorometric analysis of chlorophyll-a in the presence of chlorophyll-b and pheopigments. Limnology and Oceanography, 1994, 39: 1985-1992.

[ 8 ] Asai R, Matsukawa R, Ikebukuro K et al. Highly sensitive chemiluminescence flow-injection detection of the red tide phytoplankton Heterosigma carterae. Analytica Climica Acta, 1999, 390(1/2/3) : 237-244.

[ 9 ] Becker A, Meister A, Wilhelm C. Flow cytometric discrimination of various phycobilin-containing phytoplankton groups in a hypertrophic reservoir. Cytometry, 2002, 48(1) : 45-57.

[10 ] Stauber JL, Franklin NM, Adams MS. Applications of flow cytometry to ecotoxicity testing using microalgae. Trends Biotechnol, 2002, $20(4): 141-143$.

[11] 曹为民. 介绍一种先进的粒度测量仪器一一库尔特计数器. 电力环境保护, 1989, (1) : 50-52.

[12] Hallegraeff GF, Hara Y. Taxonomy of harmful marine raphidophytes. In: Hallegraeff GM, Anderson DM, Cerebella AD eds. Manual on harmful marine microalgae. Paris: UNESCO, 1995: 365-371.

[13] Utermöhl H. Neue wege in der quantitativan erfassung des planktons ( Mit besondere Berücksichtigung des Ultraplanktons). Verh Int Verein Theor Angew Limnol, 1931, 5: 567-595.

[14] Utermöhl H. Zur vervollkomnung der quantitativen phytoplankton methodik. Mitt Int Verein Theor Angew Limnol, 1958, 9: $1-38$.

[15] Sournia A. Phytoplankton manual. Paris: UNESCO, 1978.

[16] Paxinos R, Mitchell JG. A rapid utermöhl method for estimating algal numbers. Journal of Plankton Research, 2000,22 (12) : 2255-2262.

［17］王 瓒,乔俊莲,王国强等. 浓缩过滤——超声振荡法检测水中藻类. 中国给水排水,2008,24(14): 86-87.

[18] Steinberg MK, First MR, Lemieux EJ et al. Comparison of techniques used to count single-celled viable phytoplankton. Journal of Applied Phycology, 2012, 24(4) : 751-758.

[19] Sun J, Liu DY. Geometric models for calculating cell biovolume and surface area for phytoplankton. Journal of Plankton Research, 2003, 25(11): 1331-1346.

[20 ] Hillebrand H, Dürselen CD, Kirschtel D et al. Biovolume calculation for pelagic and benthic microalgae. Journal of Phycology, 1999, 35: 403-424.

[21] Ravi kumar MS, Ramaiah N, Tang DL. Morphometry and cell volumes of diatoms from a tropical estuary of India. India 
Journal of Marine Sciences, 2009, 38(2) : 160-165.

[22] Truby EW. Preparation of single-celled marine dinoflagellates for electron microscopy. Microscopy Research and Technique, 1997, 36 : 337-340.

[23] 陈 坤, 张前前, 史海燕等. 浮游植物计数方法比较研究. 海洋环境科学, 2007,26(4) : 383-385.

[24] Guillard RRL. Growth measurements. Division rates. In: Stein JR ed. Handbook of phycological methods. Cambridge: Cambridge University Press, 1973.

[25] Siemcki CK, Sieracki ME, Yentsch CS. An imaging-in-flow system for automated analysis of marine microplankton. Marine Ecology Progress Series, 1998, 168: 285-296.

[26] Wert EC, Dong MM, Rosario-Ortiz FL. Using digital flow cytometry to assess the degradation of three cyanobacteria species after oxidation processes. Water Research, 2013, 47(11) : 3752-3761.

[27] Eigemann F, Hilt S, Schmitt-Jansen M. Flow cytometry as a diagnostic tool for the effects of polyphenolic allelochemicals on phytoplankton. Aquatic Botany, 2013, 104: 5-14.

[28] Hyka P, Lickova S, Přibyl P et al. Flow cytometry for the development of biotechnological processes with microalgae. Biotechnology Advances, 2013, 31(1) : 2-16.

[29] Monaldi AC, Romero GG, Alanís EE et al. Digital holographic microscopy for microalgae biovolume assessment. Optics Communications, 2015, 336(1) : 255-261.

[30 ] Malkassian A, Nerini D, van Dijk MA et al. Functional analysis and classification of phytoplankton based on data from an automated flow cytometer. Cytometry A, 2011, 79: 263-275.

[31 ] Picot J, Guerin CL, le van Kim C et al. Flow cytometry: retrospective, fundamentals and recent instrumentation. Cytotechnology, 2012, 64: 109-130.

[32] Moberg EA, Sosik HM. Distance maps to estimate cell volume from two-dimensional plankton images. Limnology and Oceanography: Methods, 2012, 10: 278-288.

[33 ] Mackey DJ, Blanchot J, Higgins HW et al. Phytoplankton abundances and community structure in the equatorial Pacific. Deep Sea Research Part II, 2002, 49(13/14) : 2561-2582.

[34] Latasa M, Bidigare RR, Ondrusek ME et al. HPLC analysis of algal pigments : a comparison exercise among laboratories and recommendations for improved analytical performance. Marine Chemistry, 1996, 51(4) : 315-324.

[35 ] Wright SW, Jeffrey SW, Mantoura RFC et al. Improved HPLC method for the analysis of chlorophylls and carotenoids from marine phytoplanklon. Marine Ecology Progress Series, 1991, 77 : 183-196.

[36] Brotas V, Plantc-Cuny MR. Identification and quantification of chlorophy II and carotenoid pigments in marine sediments: A protocol for HPLC analysis. Oceanographic Acta, 1996, 19(6) : 623-634.

[37] Kwan WC, Kiln WC. HPLC pigment analysis of marine phytoplankton during a red tide occurrence in Tolo Harbour, Hong Kong. Chemosphere, 2003, 52(9) : 1633-1640.

[38 ] Zapata M, Jeffrey SW, Wright SW. Photosynthetic pigments in 37 species (65 strains) of Haptophyta: implications for oceanography and chemotaxonomy. Marine Ecology Progress Series, 2004, 270 : 83-102.

[39] 姚 鹏. 胶州湾浮游藻的色素分析和基于色素的分类方法研究 [ 学位论文]. 青岛: 中国海洋大学,2005.

[40] Hodgson DA, Wright SW, Davies N. Mass Spectrometry and reverse phase HPLC techniques for the identification of degraded fossil pigments in lake sediments and their application in palaeolimnology. Journal of Paleolimnology, 1997, 18 (4) : 335-350.

[41] Hou JJ, Huang BQ, Cao ZR et al. Effects of nutrient limitation on pigments in Thalassiosira weissflogii and Prorocentrum donghaiense. Journal of Integrative Plant Biology, 2007, 49(5) : 686-697.

[42] Catherine A, Escoffier N, Belhocine A et al. On the use of the Fluoro Probe, a phytoplankton quantification method based on fluorescence excitation spectra for large-scale surveys of lakes and reservoirs. Water Research, 2012, 46 ( 6) : 1771-1784

[43] Chang DW, Hobson P, Burch M et al. Measurement of cyanobacteria using in-vivo fluoroscopy - Effect of cyanobacterial species, pigments, and colonies. Water Research, 2012, 46(16) : 5037-5048.

[44] Medina-Cobo M, Domínguez JA, Quesada A et al. Estimation of cyanobacteria biovolume in water reservoirs by MERIS sensor. Water Research, 2014, 63: 10-20.

[45] Lunetta RS, Schaeffer BA, Stumpf RP et al. Evaluation of cyanobacteria cell count detection derived from MERIS imagery 
across the eastern USA. Remote Sensing of Environment, 2015, 157 : 24-34.

[46] Chekalyuk A, Landry M, Goericke R et al. Laser fluorescence analysis of phytoplankton across a frontal zone in the California current ecosystem. Journal of Plankton Research, 2012, 34(9) : 761-777.

[47] Lee T, Tsuzuki M, Takeuchi T et al. Quantitative determination of cyanobacteria in mixed phytoplankton assemblages by an in vivo fluorimetric method. Analytica Chimica Acta, 1995, 302(1) : 81-87.

[48 ] Beutler M, Wiltshire KH, Arp M et al. A reduced model of the fluorescence from the cyanobacterial photosynthetic apparatus designed for the in situ detection of cyanobacteria. Biochimica et Biophysica Acta, 2003, 1604(1) : 33-46.

[49] Beutler M, Wiltshire KH, Meyer B et al. A fluorometric method for the differentiation of algal populations in vivo and in situ. Photosynthesis Research, 2002, 72(1) : 39-53.

[50] 王书涛,王玉田,车仁生等. 基于小波变换的叶绿素苂光光谱测量系统研究. 应用光学,2005,26: 49-52.

[51] Chen P, Pan D, Mao Z. Application of a laser fluorometer for discriminating phytoplankton species. Optics \& Laser Technology, 2015, 67 : 50-56.

[52] Koehne B, Elli G, Jennings RC et al. Spectroscopic and molecular characterization of a long wavelength absorbing antenna of Ostreobium sp. Biochimica et Biophysica Acta, 1999, 1412(2) : 94-107.

[53 ] Carder KL, Steward RG. A remote-sensing reflectance model for red-tide dinoflagellate off West Florida. Limnology and Oceanography, 1985, 30: 286-298.

[54] Ahn YH, Shanmugam P, Ryu JH et al. Satellite detection of harmful algal bloom occurrences in Korean waters. Harmful Algae, 2006, 5(2) : 213-231.

[55 ] Brewin RJW, Hardman-Mountford NJ, Lavender SJ et al. An intercomparison of bio-optical techniques for detecting dominant phytoplankton size class from satellite remote sensing. Remote Sensing of Environment, 2011, 115(2) : 325-339.

[56] Alikas K, Kangro K, Reinart A. Detecting cyanobacterial blooms in large North European lakes using the Maximum Chlorophyll Index. Oceanologia, 2010, 52 : 237-257.

[57] Wynne TT, Stumpf RP, Tomlinson MC et al. Characterizing a cyanobacterial bloom in western Lake Erie using satellite imagery and meteorological data. Limnology and Oceanography, 2010, 55 : 2025-2036.

[58] Matthews MW, Bernard S, Robertson I. An algorithm for detecting trophic status (chlorophyll-a), cyanobacteria-dominance, surface scums and floating vegetation in inland and coastal waters. Remote Sensing of Environment, 2012, 124: $637-652$.

[59] Duan H, Ma R, Hu C. Evaluation of remote sensing algorithms for cyanobacterial pigment retrievals during spring bloom formation in several lakes of east China. Remote Sensing of Environment, 2012, 126 : 126-135.

[60 ] Hunter PD, Tyler AN, Carvalho L et al. Hyperspectral remote sensing of cyanobacterial pigments as indicators for cell populations and toxins in eutrophic lakes. Remote Sensing of Environment, 2010, 114 : 2705-2718.

[61] Ruiz-Verdú A, Simis SGH, Hoyos CD et al. An evaluation of algorithms for the remote sensing of cyanobacterial biomass. Remote Sensing of Environment, 2008, 112 : 3996-4008.

[62] Gómez JAD, Alonso CA, García AA. Remote sensing as a tool for monitoring water quality parameters for Mediterranean Lakes of European Union water framework directive( WFD) and as a system of surveillance of cyanobacterial harmful algae blooms(SCyanoHABs). Environmental Monitoring and Assessment, 2011, 181 : 317-334.

[63] de Jonge VN, Colijn F. Dynamics of microphytobenthos biomass in the Ems estuary. Marine Ecology Progress Series, 1994, 104: 185-196.

[64] Wirtz KW, Pahlow M. Dynamic chlorophyll and nitrogen: carbon regulation in algae optimizes instantaneous growth rate. Marine Ecology Progress Series, 2010, 402 : 81-96.

[65] Cho ES, Costas E. Rapid monitoring for the potentially ichthyotoxic dinoflagellate Cochlodinium polykrikoides in Korean coastal waters using fluorescent probe tools. Journal of Plankton Research, 2004, 26(2) : 175-180.

[66] Fabienne G, Laetitia P, Béatrice B et al. Monoclonal antibody against the surface of Alexandrium minutum used in a whole-cell ELISA. Harmful Algae, 2009, 8(3) : 538-545.

[67] Vrieling EG, Poll WH, Vriezekolk G et al. Immuno-flow cytometric detection of the ichthyotoxic dinoflagellates Gyrodinium aureolum and Gymnodinium nagasakiense: independence of physiological state. Journal of Sea Research, 1997, $37(1 / 2): 91-100$.

[68 ] Xin ZY, Yu ZG, Wang TC et al. Identification and quantification of the toxic dinoflagellate Gymnodinium sp. with com- 
petitive enzyme-linked immunosorbent assay(cELISA). Harmful Algae, 2005 , 4(2) : 297-307.

[69] 向军俭,凌钦婕,吕颂辉等. 四种赤潮藻多克隆抗体的制备及特异性分析. 暨南大学学报: 自然科学版, 2006,26 (5) : 700-704.

[70] Zechman FW, Zimmer EA, Theriot EC. Use of ribosomal DNA internal transcribed spacers for phylogenetic studies in diatoms. Journal of Phycology, 1994, 30: 507-512.

[71] 王 波,米铁柱,吕颂辉等. 几株原甲藻核糖体大亚基 RNA 基因的部分克隆及序列分析. 海洋学报: 中文版,2007, 29(1) : 120-126.

[72］侯建军. 赤潮生物的分子探针检测方法及其应用研究 [学位论文]. 厦门:厦门大学,2005.

[73 ] Steffen MM, Zhu Z, McKay RML et al. Taxonomic assessment of a toxic cyanobacteria shift in hypereutrophic Grand Lake St. Marys( Ohio, USA). Harmful Algae, 2014, 33: 12-18.

[74] Dueymes C, Decout JL, Peltie P et al. Fluorescent deazaitavin oligonucleotide probes for selective detection of DNA. Angewandte Chemie International Edition, 2002, 41(3) : 486-489.

[75] Amann R, Ludwig W, Ribosomal RNA - targeted nucleic acid probes for studies in microbial ecology. FEMS Microbiology Reviews, 2000, 24(5) : 555-565.

[76] Kim CJ, Kim CH, Sako Y. Development of molecular identification method for genus Alexandrium (Dinophyceae) using whole-cell FISH. Marine Biotechnology, 2005, 7(3) : 215-222.

[77] Miller PE, Scholin CA. On detection of Pseudo-nitzschia (Baeillariophyceae) species using whole cell hybridization: Sample fixation and stability. Journal of Phycology, 2000, 36 : 238-250.

[78 ] Hosoi-Tanabe S, Sako Y. Rapid detection of natural ceils of Alexandrium tamarense and A. catenella (Dinophyceae) by fluorescence in situ hybridization. Harmful Algae, 2005, 4 : 319-328.

[79] Tengs T, Bowers HA, Ziman AP et al. Genetic polymorphism in Gymnodinium galatheanum chloroplast DNA sequences and development of a molecular detection assay. Melecular Ecology, 2001, 10 : 515-523.

[80] He SY, Yu ZG. A real-time PCR method for rapid detection of Gymnodinium sanguineum. Journal of Zhejiang University: Agric \& Life Sci, 2009, 35(2) : 119-126.

[81] Cai QS, Li RX, Zhen Y et al. Detection of two Prorocentrum species using sandwich hybridization integrated with nuclease protection assay. Harmful Algae, 2006, 5 (3) : 300-309.

[82] 朱 霞. 应用电致化学发光分子探针技术对微小原甲藻的检测 [ 学位论文]. 青岛: 中国海洋大学,2011.

[83］董正榛,董振芳,丁德文. 快速测定藻生物量的方法探讨. 实验与技术,2004,28(11): 1-5.

[84] 胡先文,董元彦,张新萍等. 可见分光光度法测定水华鱼腥藻. 华中农业大学学报,2002,21(3) : 295-297.

[85］陈纬栋,王 崇,胡晓芳等. 应用苂光分析技术检测蓝藻生物量. 净水技术,2010,29(6) : 80-84.

[86] 杨晓冬. 浅析苂光法测定蓝藻生物量的可行性. 环境科学导刊,2011,30(5): 89-91.

[87] 沈萍萍, 李朝晖, 齐雨藻等. 光密度法测定微藻生物量. 暨南大学学报: 自然科学版,2001, (3): 115-119.

[88］侯建军,黄邦钦,戴相辉. 赤潮藻细胞计数方法比较研究. 中国公共卫生,2004,20(8): 907-910.

[89］周永欣,章宗涉. 水生生物毒性试验方法. 北京:农业出版社,1998: 170-190.

[90］张 奇. 库尔特微粒分析仪颗粒测试方法的探讨. 分析测试学报,1994,13(4) : 42-44.

[91] Popp BN, Laws EA, Bidigare RR et al. The effect of phytoplankton cell geometry on carbon isotopic-fractionation. Geochinica et Cosmochimica Acta, 1998, 62(1) : 69-77.

[92] 彦昌敬. 植物组织培养手册. 上海: 上海科学技术出版社, 1990: 453.

[93 ] Brussaard C, Thyrhaug R, Dominigue M et al. Flow cytometric analyses of viral infection in two marine phytoplankton species, Micromonas pusilla( Prasinophyceae) and Phaeocystis pouchetii (Prymnesiophyceae). Journal of Phycology, 1999, $35(5)$ : 941-948.

[94] 张利华,张 经, 昆 敏. 流式细胞术对微微型浮游植物识别初探. 海洋科学, 2002,26(3): 60-65.

[95 ] Montagnes DJS, Berges JA, Harrison PJ et al. Estimating carbon, nitrogen, protein, and chlorophyll a from volume in marine phytoplankton. Limnology and Oceanography, 1994, 39(5) : 1044-1060.

［96］杨茹君,王修林,石晓勇等. 海洋浮游植物粒径分布方法研究. 高技术通讯,2004,14(6) : 89-94. 